Annals of Pure and Applied Mathematics

Vol. 12, No. 2, 2016, 185-195

ISSN: 2279-087X (P), 2279-0888(online)

Published on 23 November 2016

www.researchmathsci.org

DOI: http://dx.doi.org/10.22457/apam.v12n2a10

Annals of

Pure and Applied

Mathematics

\title{
A New Approach Towards Laplace Homotopy Perturbation Method
}

\author{
Chandrali Baishya \\ Department of Studies and Research in Mathematics \\ Tumkur University, B H Road, Tumkur -572103, Karnataka, India \\ email: baishyachandrali@gmail.com
}

Received 30 October 2016; accepted 19 November 2016

\begin{abstract}
Laplace Homotopy Perturbation method is projected in a modified form to solve highly nonlinear differential equations.This approach simplifies the equations and avoid the computations of Laplace Transforms of complicated functions, which in turn save the computation time of the computational tools. The results are compared with results obtained by using reduced differential transform method.
\end{abstract}

Keywords: Laplace Transforms, Homotopy Perturbation Mathod, Reduced Differential Transform Mathod.

AMS Mathematics Subject Classification (2010): 35B20, 35K55, 35L70, 44A10

\section{Introduction}

The rapid developments in science and technology have given birth to many complex nonlinear problems in various fields of studies viz. solid state physics, plasma physics, fluid mechanics, biology, chemistry etc.Studies of these highly nonlinear problems demand the emerge of many analytical as well as numerical and approximation techniques and continuous approach to modify them. In the recent years, many remarkable semi-analytic methods are contributed to solve nonlinear problems. Adomain Decomposition Method [1-3], Variational Iteration Method [4-5], Homotopy Perturbation Method (HPM) [6-7], Differential Transform Method (DTM) [12-15], Sine-Cosine method [8-9],Tanh Function mathod [10] and many other numerical methods [18-19] used to solve such problems.The key advantage of these semi-analytic methods are that the complicated nonlinear problems can be solved without using any transformation, linearization, discretization or any other constraints.In this work, our approach deals with Laplace Homotopy Perturbation Method (LHPM) and Reduced Differential Transform Method (RDTM). DTM was introduced by Zhou to solve linear and nonlinear initial value problems of electric circuit analysis and modified by Keskin as RDTM. He developed the HPM for solving linear, nonlinear initial and boundary value problems. HPM combining with Laplace Transformation (LT) has been introduced by Khan and Wu for solving various linear and nonlinear equations and proved to be very efficient due to the application of LT. But this method involves the computation of LT of complicated function, for which the computational tool like Mathematica consumes lots of computation time to produce output.To overcome this disadvantage, in this work, we have presented the 


\section{Chandrali Baishya}

LHPM [16-17] in a new form. The approach is applied to highly nonlinear diffusion and wave equations [11] involving logarithmic and exponential nonlinearity and obtained satisfactory results. Even for third order time derivative PDE the approach is equally efficient. The obtained results are verified with the help of RDTM.

\section{Modified laplace homotopy perturbation method (MLHPM)}

We consider a general nonlinear partial differential equation with initial conditions of the form

$$
D u(x, t)+R u(x, t)+N u(x, t)=g(x, t)
$$

with initial conditions

$$
\frac{\partial^{i} u(x, 0)}{\partial t^{i}}=f_{i}(x), \quad i=0,1,2, \ldots m-1
$$

where $\mathrm{D}$ is the $\mathrm{mth}$ order linear differential operator $D=\frac{\partial^{m}}{\partial t^{m}}, \mathrm{R}$ is the linear differential operator, $\mathrm{N}$ represents the general nonlinear differential operator and $\mathrm{g}(\mathrm{x}, \mathrm{t})$ is the source term. Taking Laplace transform (let us denote it by L) on both sides of eqquation (1), we get

$$
L[D u(x, t)]+L[R u(x, t)]+L[N u(x, t)]=L[g(x, t)]
$$

Using differential property of Laplace transform, we have

$$
L[u(x, t)]=\sum_{i=0}^{m-1} \frac{f_{i}(x)}{s^{(i+1)}}-\frac{1}{s^{m}} L[R u(x, t)+N u(x, t)-g(x, t)]
$$

Operating with the Laplace inverse transform on both sides of equation (3), gives

$$
u(x, t)=\sum_{i=0}^{m-1} f_{i}(x) \frac{t^{i}}{i !}-L^{-1}\left[\frac{1}{s^{m}} L[R u(x, t)+N u(x, t)-g(x, t)]\right.
$$

In the He's HPM, the basic assumption is that the solution can be represented in the form of

$$
u(x, t)=\sum_{n=0}^{\infty} p^{n} u_{n}(x, t)
$$

and the nonlinear term may be represented as

$$
N u(x, t)=\sum_{n=0}^{\infty} p^{n} H_{n}(u)
$$

where $H_{n}(u)$ may be represented by

$$
H_{n}\left(u_{0}, u_{1}, \ldots u_{n}\right)=\frac{1}{n !} \frac{\partial^{n}}{\partial p^{n}}\left[N\left(\sum_{i=0}^{n} p^{i} u_{i}(x, t)\right]\right.
$$

Using equation (5) and equation (6) in equation (4), in MLHPM, we construct the homotopy as

$$
u(x, t)=\sum_{i=0}^{m-1} p^{i} f_{i}(x) \frac{t^{i}}{i !}+p^{m}\left[g(x, t)-L^{-1}\left[\frac{1}{s^{m}} L[R u(x, t)+N u(x, t)]\right]\right.
$$

Comparing the coefficients of like power of $p$ in euation (8), we find the values of 
A New Approach Towards Laplace Homotopy Perturbation Method $u_{0}(x, t), u_{1}(x, t), u_{2}(x, t) \ldots$ and so on. Finally the analytic solution $u(x, t)$ is approximated by the truncated series as follows:

$$
u(x, t)=\lim _{N \rightarrow \infty}\left(\sum_{n=0}^{N} u_{n}(x, t)\right)
$$

This is the MLHPM.

\section{Reduced differential transform method}

Consider a function of two variable $u(x, t)$ and suppose that it can be represented as a product of two single valued functions i.e. $u(x, t)=f(x) g(t)$. Based on the property of one dimensional differential transform, the function $u(x, t)$ can be represented as follows:

$$
u(x, t)=\left(\sum_{i=0}^{\infty} F(i) x^{i}\right)\left(\sum_{j=0}^{\infty} G(j) t^{j}\right)=\sum_{k=0}^{\infty} U_{k}(x) t^{k}
$$

where $U_{k}(x)$ is called t-dimensional spectrum function of $u(x, t)$.

Definition 3.1. Let $u(x, t)$ be the original analytic function and differentiated continuously in the domain of interest. Then let

$$
U_{k}(x)=\frac{1}{k !}\left[\frac{\partial^{k}}{\partial t^{k}} u(x, t)\right]_{t=0}
$$

The spectrum $U_{k}(x)$ is the transformed or T-function.

Definition 3.2. The differential inverse function is defined as follows:

$$
u(x, t)=\sum_{k=0}^{\infty} U_{k}(x) t^{k}
$$

Combining (10) and (11 we may write

$$
u(x, t)=\sum_{k=0}^{\infty} \frac{1}{k !}\left[\frac{\partial^{k}}{\partial t^{k}} u(x, t)\right]_{t=0} t^{k}
$$

To illustrate the basic concepts of the RDTM, consider the nonlinear PDE (1). According to RDTM, we can construct the following iteration formula:

$$
(k+1)(k+2) \ldots(k+m) U_{k+m}(x)=G_{k}(x)-R U_{k}(x)-N U_{k}(x)
$$

with initial condition

$$
U_{k}(x)=f_{k}(x)
$$

Substituting (14) into (13) and then by iteration we obtain the following values of $U_{k}(x)$. Then, the inverse transformation of the set of values $\left\{U_{k}(x)\right\}_{k=0}^{n}$ gives the $\mathrm{n}$-term approximation to solution as follows: 
Chandrali Baishya

$$
u_{n}(x, t)=\sum_{k=0}^{n} U_{k}(x) t^{k}
$$

Therefore the exact solution of the problem is given by

$$
u(x, t)=\lim _{n \rightarrow \infty} u_{n}(x, t)
$$

Some fundamental transformations, which can be readily obtained are listed in the following table.

$$
\begin{array}{ll}
\begin{array}{ll}
\text { Original form } \\
u(x, t)=w(x, t) \pm v(x, t)
\end{array} & \begin{array}{l}
\text { Transformed form } \\
U_{k}(x)=W_{k}(x) \pm V_{k}(x)
\end{array} \\
u(x, t)=\alpha w(x, t) & U_{k}(x)=\alpha W_{k}(x) \\
u(x, t)=\frac{\partial^{m}}{\partial t^{m}} w(x, t) & U_{k}(x)=\frac{(k+m) !}{k !} W_{k+m}(x) \\
u(x, t)=\frac{\partial}{\partial x} w(x, t) & U_{k}(x)=\frac{\partial}{\partial x} W_{k}(x) \\
u(x, t)=x^{m} t^{n} & U_{k}(x)=x^{m} \delta(k-n) \text { where } \\
u(x, t)=x^{m} t^{n} w(x, t) & \delta(k-n)=\left\{\begin{array}{cc}
1 & \text { if } \mathrm{k}=\mathrm{n} \\
0 & \text { otherwise }
\end{array}\right. \\
u(x, t)=x^{m} t^{n} w(x, t) & U_{k}(x)=x^{m} W_{k-n}(x)
\end{array}
$$

Table 1: Fundamental Operations in RDTM

Theorem 3.1. If $g(x, t)=u(x, t) \ln u(x, t), u(x, 0)=f(x)$ and $G_{k}(x)$ is the reduced differential transform( RDT) of the function $g(x, t)$ then

$$
\begin{aligned}
& G_{0}(x)=U_{0}(x) \ln U_{0}(x) \\
& G_{k+1}(x)=\frac{1}{(k+1) U_{0}(x)} \sum_{i=0}^{k}\left[(k-i+1) U_{i}(x) U_{k-i+1}(x)+(k-2 i+1) G_{i}(x) U_{k-i+1}(x)\right], \\
& k \geq 0 .
\end{aligned}
$$

Theorem 3.2. If $g(x, t)=e^{a u(x, t)}, u(x, 0)=f(x)$ and $G_{k}(x)$ is the RDT of the function $g(x, t)$ then

$$
\begin{aligned}
& G_{0}(x)=e^{a U_{0}(x)} \\
& G_{k}(x)=\frac{a}{k} \sum_{i=1}^{k} i U_{i}(x) G_{k-i}(x), k \geq 1
\end{aligned}
$$

\section{Numerical examples}

Example 4.1. Consider the equation

$$
u_{t}(x, t)-x u_{x x}(x, t)=u(x, t) \ln u(x, t)
$$


A New Approach Towards Laplace Homotopy Perturbation Method with initial condition

$$
u(x, 0)=e^{x}
$$

(i) By MLHPM

Taking Laplace transform on both sides of the equation (17) we obtained

$$
L[u(x, t)]=\frac{e^{x}}{s}-\frac{1}{s} L\left[x u_{x x}(x, t)+u(x, t) \ln u(x, t)\right]
$$

Operating with the Laplace inverse transform on both sides of equation (19), gives

$$
u(x, t)=e^{x}-L^{-1}\left[\frac{1}{S} L\left[x u_{x x}(x, t)+u(x, t) \ln u(x, t)\right]\right]
$$

According to He's HPM, the basic solution is represented by equation (5) and the nonlinear term is represented by equations (6) and (7).Therefore homotopy of equation (20) becomes

$$
\sum_{n=0}^{\infty} p^{n} u_{n}(x, t)=e^{x}-p L^{-1}\left[\frac{1}{s} L\left[x \sum_{n=0}^{\infty} p^{n} u_{n, x x}(x, t)+\sum_{n=0}^{\infty} p^{n} H_{n}(u)\right]\right]
$$

where $p \varepsilon[0,1]$ is the embedding parameter and $H_{n}(u)$ is the He's polynomial represented by

$$
\begin{aligned}
& H_{n}\left(u_{0}, u_{1}, \ldots u_{n}\right)=\frac{1}{n !} \frac{\partial^{n}}{\partial p^{n}}\left[\sum_{i=0}^{n} p^{i} u_{i}(x, t) \ln \left(\sum_{i=0}^{n} p^{i} u_{i}(x, t)\right)\right] \\
& =\frac{1}{n !} \frac{\partial^{n}}{\partial p^{n}} \sum_{i=0}^{n} p^{i} u_{i}(x, t)\left[\ln u_{0}(x, t)+\ln \left(1+\sum_{i=1}^{n} p^{i} \frac{u_{i}(x, t)}{u_{0}(x, t)}\right)\right] \\
& \left.=\frac{1}{n !} \frac{\partial^{n}}{\partial p^{n}} \sum_{i=0}^{n} p^{i} u_{i}(x, t)\left[\ln u_{0}(x, t)+\sum_{j=1}^{\infty}(-1)^{j+1} \frac{1}{j}\left(\sum_{i=1}^{n} p^{i} \frac{u_{i}(x, t)}{u_{0}(x, t)}\right)^{j}\right)\right]
\end{aligned}
$$

Comparing the like powers of $p$ in equation (21) the following approximations are obtained:

$$
\begin{aligned}
& p^{0}: u_{0}(x, t)=e^{x} \\
& p^{1}: u_{1}=L^{-1}\left[\frac{1}{s} L\left[x u_{0, x x}(x, t)+H_{0}(u)\right]\right]=2 t e^{x} x \\
& p^{2}: u_{2}=L^{-1}\left[\frac{1}{S} L\left[x u_{1, x x}(x, t)+H_{1}(u)\right]\right]=t^{2} e^{x}\left(2 x^{2}+3 x\right) \\
& p^{3}: u_{3}=L^{-1}\left[\frac{1}{S} L\left[x u_{2, x x}(x, t)+H_{2}(u)\right]\right]=\frac{1}{3} t^{3} e^{x} x\left(4 x^{2}+18 x+13\right) \\
& p^{4}: u_{4}=L^{-1}\left[\frac{1}{S} L\left[x u_{3, x x}(x, t)+H_{3}(u)\right]\right]=\frac{1}{12} t^{4} e^{x} x\left(8 x^{3}+72 x^{2}+158 x+75\right) \\
& p^{5}: u_{5}=\frac{1}{60} t^{5} e^{x} x\left(16 x^{4}+240 x^{3}+1060 x^{2}+1530 x+541\right) \\
& p^{6}: u_{6}=\frac{1}{360} t^{6} e^{x} x\left(32 x^{5}+720 x^{4}+5320 x^{3}+15480 x^{2}+16622 x+4683\right.
\end{aligned}
$$


Chandrali Baishya

$+201978 x+47293)$

$$
\begin{aligned}
p^{7}: u_{7} & =\frac{1}{2520} t^{7} e^{x} x\left(64 x^{6}+5856 x^{5}+22400 x^{4}+109200 x^{3}+236404 x^{2}\right) \\
p^{8}: u_{8}= & \frac{1}{20160} t^{8} e^{x} x\left(128 x^{7}+182016 x^{6}+375616 x^{5}+730080 x^{4}+2231208 x^{3}\right. \\
& \left.+3841992 x^{2}+2724878 x+545835\right)
\end{aligned}
$$

Solution is represented using the truncated series (9) as

$$
u(x, t)=u_{0}+u_{1}+u_{2}+u_{3}+u_{4}+\ldots . .
$$

(ii) By RDTM

According to the RDTM and Table 1 the differential transform of equations (17) and (18) are as follows:

$$
\begin{gathered}
(k+1) U_{k+1}(x)-x \frac{\partial^{2}}{\partial x^{2}} U_{k}(x)=G_{k}(x) \\
\text { with } U_{0}(x)=e^{x}
\end{gathered}
$$

here $G_{k}(x)$ represents the RDT of $u(x, t) \ln u(x, t)$. Clearly $G_{0}(x)=x e^{x}$. Using Theorem 3 into equation (22) we obtain

$$
\begin{aligned}
& U_{k+1}(x)=\frac{1}{k+1}\left[x \frac{\partial^{2}}{\partial x^{2}} U_{k}(x)\right. \\
& \left.+\frac{1}{k U_{0}(x)} \sum_{i=0}^{k-1} U_{k-i}(x)\left\{(k-i) U_{i}(x)+(k-2 i) G_{i}(x)\right\}\right]
\end{aligned}
$$

where $k \geq 1$.

Equation (24) in tern implies

$$
\begin{aligned}
& U_{k+1}(x)=\frac{1}{k+1}\left[x \frac{\partial^{2}}{\partial x^{2}} U_{k}(x)+\frac{1}{k U_{0}(x)} \sum_{i=0}^{k-1} U_{k-i}(x)\left\{(k-i) U_{i}(x)\right.\right. \\
& \left.\left.+(k-2 i)\left\{(i+1) U_{i+1}(x)-x \frac{\partial^{2}}{\partial x^{2}} U_{i}(x)\right\}\right\}\right]
\end{aligned}
$$

From equation (22) we obtain

$$
U_{1}(x)=2 x e^{x}
$$

Using (25) as an iteration formula we obtain the following values of $U_{k}(x)$

$$
\begin{aligned}
& U_{2}=\frac{1}{2} e^{x}\left(4 x^{2}+6 x\right) \\
& U_{3}(x)=\frac{1}{3} e^{x}\left(4 x^{3}+18 x^{2}+13 x\right) \\
& U_{4}(x)=\frac{1}{4} e^{x}\left(\frac{8 x^{4}}{3}+24 x^{3}+\frac{158 x^{2}}{3}+25 x\right)
\end{aligned}
$$


A New Approach Towards Laplace Homotopy Perturbation Method

$$
\begin{aligned}
U_{5}(x) & =\frac{1}{5} e^{x}\left(\frac{4 x^{5}}{3}+20 x^{4}+\frac{265 x^{3}}{3}+\frac{255 x^{2}}{2}+\frac{541 x}{12}\right) \\
U_{6}(x)= & \frac{1}{360} e^{x} x\left(32 x^{5}+720 x^{4}+5320 x^{3}+15480 x^{2}+16622 x+4683\right) \\
U_{7}(x)= & \frac{1}{2520} e^{x} x\left(64 x^{6}+2016 x^{5}+22400 x^{4}+109200 x^{3}+236404 x^{2}\right. \\
& +201978 x+47293)
\end{aligned}
$$

and so on. Using inverse transform (11) and (12) we obtain the solution of (17) as

$$
\begin{aligned}
u(x, t)=e^{x}[1+ & 2 x t+\frac{1}{2} t^{2}\left(6 x+4 x^{2}\right)+\frac{1}{3} t^{3}\left(13 x+24 x^{2}+8 x^{3}\right) \\
& +\frac{1}{4} t^{4}\left(29 x+\left(212 x^{2}\right) / 3+\left(100 x^{3}\right) / 3+\left(8 x^{4}\right) / 3\right)+\ldots
\end{aligned}
$$

\section{Example 4.2. Consider the equation}

$$
u_{t t}(x, t)-x^{2} u_{x x}(x, t)=a+b e^{\lambda u(x, t)}
$$

with initial condition

(i) By MLHPM

$$
u(x, 0)=x, u_{t}(x, 0)=x^{2}
$$

By taking laplace transform and then inverse Laplace transform, using we can construct the homotopy of the equation (26) as

$$
\sum_{n=0}^{\infty} p^{n} u_{n}(x, t)=x+p x^{2} t+p^{2}\left(L^{-1}\left[\frac{1}{s^{2}} L\left[x^{2} \sum_{n=0}^{\infty} p^{n} u_{n, x x}+b \sum_{n=0}^{\infty} p^{n} H_{n}(u)\right]\right]+\frac{a t^{2}}{2}\right)
$$

where solution is represented by equation (5) and nonlinear term is represented by He's polynomial as

$$
H_{n}\left(u_{0}, u_{1}, \ldots u_{n}\right)=\frac{1}{n !} \frac{\partial^{n}}{\partial p^{n}} e^{\lambda \sum_{i=0}^{n} p^{i} u_{i}(x, t)}, p=0
$$

Comparing the like power in $p$ in equation (28) the following approximations are obtained:

$$
\begin{aligned}
& p^{0}: u_{0}(x, t)=x \\
& p^{1}: u_{1}=t x^{2} \\
& p^{2}: u_{2}=L^{-1}\left[\frac{1}{s^{2}} L\left[x^{2} u_{0, x x}(x, t)+b H_{0}(u)\right]\right]+\frac{a t^{2}}{2}=\frac{t^{2}}{2}\left(a+b e^{\lambda x}\right) \\
& p^{3}: u_{3}=L^{-1}\left[\frac{1}{s^{2}} L\left[x^{2} u_{1, x x}(x, t)+b H_{1}(u)\right]\right]=\frac{1}{6} t^{3} x^{2}\left(b \lambda e^{\lambda x}+2\right)
\end{aligned}
$$


Chandrali Baishya

$$
\begin{aligned}
p^{4}: u_{4}= & L^{-1}\left[\frac{1}{s^{2}} L\left[x^{2} u_{2, x x}(x, t)+b H_{2}(u)\right]\right]=\frac{1}{24} t^{4} e^{\lambda x}\left(a b \lambda+b \lambda^{2} x^{4}+b \lambda^{2} x^{2}\right)+\frac{1}{24} b^{2} \lambda t^{4} e^{2 \lambda x} \\
p^{5}: u_{5}= & \frac{1}{120} e^{\lambda x}\left(3 a b \lambda^{2} t^{5} x^{2}+b \lambda^{3} t^{5} x^{6}+b \lambda^{3} t^{5} x^{4}+4 b \lambda^{2} t^{5} x^{3}+4 b \lambda t^{5} x^{2}\right) \\
& +\frac{1}{30} b^{2} \lambda^{2} t^{5} x^{2} e^{2 \lambda x}+\frac{t^{5} x^{2}}{30} \\
p^{6}: u_{6}= & \frac{1}{720} b \lambda^{2} t^{6} e^{\lambda x}\left(3 a^{2}+a\left(7 b e^{\lambda x}+6 \lambda x^{4}\right)+4 b^{2} e^{2 \lambda x}+b \lambda\left(11 x^{2}+1\right) x^{2} e^{\lambda x}\right. \\
+ & \left.x^{4}\left(\lambda^{2} x^{4}+8\right)\right)+\frac{1}{720} b \lambda^{2} t^{6} x^{2} e^{\lambda x}\left(a \lambda+4 b \lambda e^{\lambda x}+\lambda^{2} x^{4}+8 \lambda x^{3}+\lambda^{2} x^{2}+12 x^{2}+4 \lambda x+2\right)
\end{aligned}
$$

Solution is represented using the truncated series (9) as

(ii) By RDTM

$$
u(x, t)=u_{0}+u_{1}+u_{2}+u_{3}+u_{4}+\ldots . .
$$

According to the RDTM and Table 1 the differential transform of equation (26) and (27) are as follows:

$$
(k+1)(k+2) U_{k+2}(x)-x^{2} \frac{\partial^{2}}{\partial x^{2}} U_{k}(x)=a \delta(k)+b G_{k}(x)
$$

$G_{k}(x)$ is the differential transform of $e^{\lambda u(x, t)}$.

and

$$
U_{0}(x)=x, U_{1}(x)=x^{2}
$$

Equation (26), by the influence of Theorem 4 may be written as

$U_{k+2}(x)=\frac{1}{(k+1)(k+2)}\left[x^{2} \frac{\partial^{2}}{\partial x^{2}} U_{k}(x)+a \delta(k)+\frac{b \lambda}{k} \sum_{i=1}^{k} i U_{i}(x) G_{k-i}(x)\right]$

Also from Theorem 4,

and

$$
G_{0}(x)=e^{\lambda x}
$$

From (26):

$$
G_{1}(x)=\lambda x^{2} e^{\lambda x}
$$

$$
U_{2}(x)=\frac{1}{2}\left(a+b e^{\lambda x}\right)
$$

Using equation (32) as iteration formula, the following values of $U_{k}(x)$ are obtained:

$U_{3}(x)=\frac{1}{6} x^{2}\left(b \lambda e^{\lambda x}+2\right)$

$U_{4}(x)=\frac{1}{12} e^{\lambda x}\left(\frac{a b \lambda}{2}+\frac{1}{2} b \lambda^{2} x^{4}+\frac{1}{2} b \lambda^{2} x^{2}\right)+\frac{1}{24} b^{2} \lambda e^{2 \lambda x}$ 
A New Approach Towards Laplace Homotopy Perturbation Method

$$
\begin{aligned}
& U_{5}(x)=\frac{1}{120} e^{\lambda x}\left(3 a b \lambda^{2} x^{2}+b \lambda^{3} x^{6}+b \lambda^{3} x^{4}+4 b \lambda^{2} x^{3}+4 b \lambda x^{2}\right)+\frac{1}{30} b^{2} \lambda^{2} x^{2} e^{2 \lambda x}+\frac{x^{2}}{30} \\
& U_{6}(x)=\frac{1}{720} b \lambda^{2} e^{\lambda x}\left(3 a^{2}+a\left(7 b e^{\lambda x}+6 \lambda x^{4}\right)+4 b^{2} e^{2 \lambda x}+b \lambda\left(11 x^{2}+1\right) x^{2} e^{\lambda x}\right. \\
& \left.+x^{4}\left(\lambda^{2} x^{4}+8\right)\right)+\frac{1}{720} b \lambda^{2} x^{2} e^{\lambda x}\left(a \lambda+4 b \lambda e^{\lambda x}+\lambda^{2} x^{4}+8 \lambda x^{3}+\lambda^{2} x^{2}+12 x^{2}+4 \lambda x+2\right)
\end{aligned}
$$

and so on. The solution is obtained by using the equation (15) and (16). We observe that solution obtained by both the methods are exacly same.

\section{Example 4.3. Consider the equation}

$$
u_{t t t}(x, t)-u_{x x}(x, t)=e^{t}+e^{u(x, t)}
$$

with initial condition

$$
u(x, 0)=x, \quad u_{t}(x, 0)=x^{2}, \quad u_{t t}(x, 0)=x^{2}
$$

\section{(i) By MLHPM}

By taking laplace transform and then inverse Laplace transform, using we can construct the homotopy of the equation as

$$
\sum_{n=0}^{\infty} p^{n} u_{n}(x, t)=x+p x^{2} t+\frac{1}{2} p^{2} x^{3} t^{2}+p^{3}\left(L^{-1}\left[\frac{1}{s^{2}} L\left[x^{2} \sum_{n=0}^{\infty} p^{n} u_{n, x x}+\sum_{n=0}^{\infty} p^{n} H_{n}(u)+e^{t}\right]\right]\right)
$$

where solution is represented by equation (5) and nonlinear term is represented by He's polynomial as

$$
H_{n}\left(u_{0}, u_{1}, \ldots u_{n}\right)=\frac{1}{n !} \frac{\partial^{n}}{\partial p^{n}} e^{\lambda \sum_{i=0}^{n} p^{i_{i}(x, t)}}, p=0
$$

Comparing the like power in $p$ in equation (36) the following approximations to the solution is obtained:

$$
\begin{aligned}
u(x, t)=t^{6}\left(\frac{e^{x} x^{6}}{720}\right. & \left.+\frac{e^{x} x^{5}}{120}+\frac{e^{x}}{720}+\frac{e^{2 x}}{720}\right)+t^{5}\left(\frac{e^{x} x^{4}}{120}+\frac{e^{x} x^{3}}{120}+\frac{x}{20}-\frac{e^{x}}{120}\right) \\
& +t^{4}\left(\frac{e^{x} x^{2}}{24}-\frac{e^{x}}{24}+\frac{1}{12}\right)+\frac{t^{3} e^{x}}{3}+t^{2}\left(\frac{x^{3}}{2}-\frac{e^{x}}{2}-\frac{1}{2}\right)+t\left(x^{2}-e^{x}-1\right) \\
& +e^{t+x}+e^{t}-e^{x}+x+1+\ldots
\end{aligned}
$$

(ii) By RDTM

According to the RDTM and Table 1 the differential transform of equation (34) and (35) are as follows:

$$
(k+1)(k+2)(k+3) U_{k+3}(x)-\frac{\partial^{2}}{\partial x^{2}} U_{k}(x)=\frac{1}{k !}+G_{k}(x)
$$

$G_{k}(x)$ is the differential transform of $e^{u(x, t)}$.

and 
Chandrali Baishya

$$
U_{0}(x)=x, U_{1}(x)=x^{2}, U_{2}=x^{3}
$$

Equation (38), by the influence of Theorem 4 may be written as

$$
U_{k+3}(x)=\frac{1}{(k+1)(k+2)(k+3)}\left[\frac{\partial^{2}}{\partial x^{2}} U_{k}(x)+\frac{1}{k !}+\frac{1}{k} \sum_{i=1}^{k} i U_{i}(x) G_{k-i}(x)\right]
$$

Using equation (40) as an iteration formula the solution is obtained as

$$
\begin{gathered}
u(x, t)=\frac{1}{720} t^{6}\left(e^{x}\left(x^{6}+6 x^{5}+2\right)+e^{2 x}+1\right)+\frac{1}{120} t^{5}\left(e^{x} x^{4}+2 e^{x} x^{3}+12 x+1\right) \\
+\frac{1}{24} t^{4}\left(e^{x} x^{2}+3\right)+\frac{1}{6} t^{3}\left(e^{x}+1\right)+t^{2} x^{3}+t x^{2}+x+\ldots
\end{gathered}
$$

Since the direct comparision of the two methods MLHPM and RDTM is not visible here, we shall compare them graphically:

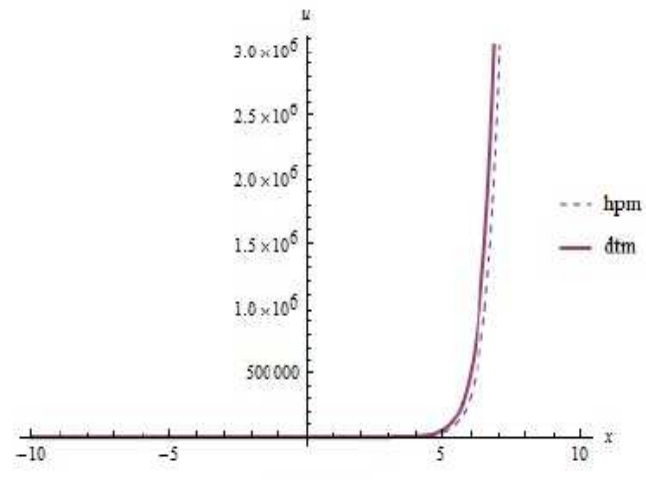

(A) $\mathrm{t}=1$

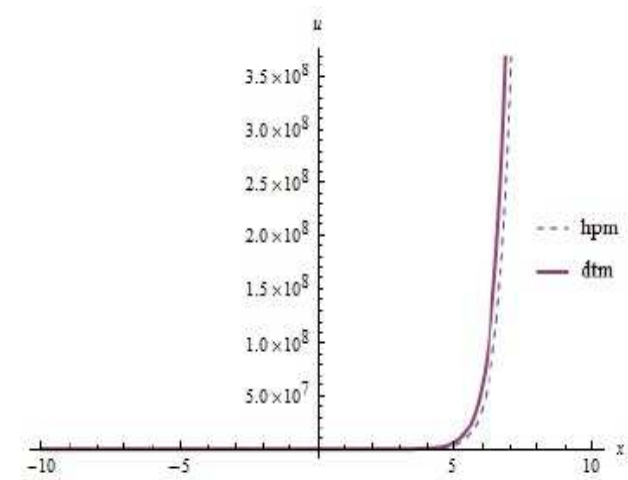

(B) $\mathrm{t}=3$

Figure 1:

\section{Conclusion}

In this work, nonlinear PDE with variable coefficient involving logarithmic and exponential nonlinearity are solved by MLHPM. Had the traditional LHPM been applied, then from equation (1), the homotopy would have been constructed as follows:

$$
u(x, t)=\sum_{i=0}^{m-1} f_{i}(x) \frac{t^{i}}{i !}+p\left[L^{-1}\left[\frac{1}{s^{m}} L[g(x, t)-R u(x, t)+N u(x, t)]\right]\right.
$$

Even for an equation involving second order time derivative, this leads to the tedious computations of Laplace Transform. But MLHPM makes the computation very simple. In this paper, the results obtained by MLHPM are verified by using RDTM.

\section{REFERENCES}

1. G.Adomian, Solving Frontier Problems of Physics: The Decomposition Method, Kluwer Acad. Publ., Dordrecht, 1994.

2. G.Adomian, A review of the decomposition method and some recent results for nonlinear equations, Comput. Math. Appl., 21(1991) 101-127. 
A New Approach Towards Laplace Homotopy Perturbation Method

3. R.Rach, A bibliography of the theory and applications of the Adomian decomposition method, Kybernetes, 41(2012) 1087-1148.

4. J.H.He, Variational iteration method- a kind of non-linear analytic technique: some examples, International Journal of Non-linear Mechanics, 34(4) (1999) 699-708.

5. A.T.Abassy, M.A.El-Tawil, H.El. Zoheirt, Solving nonlinear partial differential equations using the midified variational iteration pade technique, Journal of Computational and Applied Mathematics, 207(1) (2007) 73-91.

6. J.H.He, Homotopy perturbation method: a new non-linear technique, Applied Mathematics and Computation, 135 (2003) 73-79.

7. S.J.Liao, On the homotopy analysis method for nonlinear problems, Applied Mathematics and Computation, 147(2) (2004) 499-513.

8. A.M.Wazwaz, A sine-cosine method for handling nonlinear wave equations, Mathematical and Computer Modeling, 40 (2004) 499-508.

9. A.M.Wazwaz, The tanh and sine cosine methods for the complex modified and generalized KdV equations, Computers and Mathematics with Applications, 49 (2005) 1101-1112.

10. D.J.Evans and K.R.Raslan, The Tanh-Function method for solving some important nonlinear partial differential equation, International Journal of Computer Mathematics, 82 (2005) 897-905.

11. A.M.Wazwaz, Handbook of Differential Equations: Evolutionary Equations 4, Elsevier, (2008) 485-568.

12. Y.Keskin and G.Oturancç, The reduced differential transform method for partial differential equations, Int. J. Nonlin. Sci. Numer. Simul., 10 (6) (2009) 741-749.

13. Y.Keskin and G.Oturanç, Reduced differential transform method for generalized KdV equations, Mathematical and Computational Applications, 15(3) (2010) 382-392.

14. Y.Keskin and G.Oturanç, The reduced differential transform method for solving linear and nonlinear wave equations, Iran. J. Sci. Technol., 34 (2) (2010) 113-122.

15. A.Sarvanan and N.Magesh, A comparision between the reduced differential transform method and adomain decomposition method for the newell-whitehead-segel equation, Journal of Egyptian Mathematical Society, 21(3) (2013) 259-265.

16. M.Madani and M.Fatizadeh, Homotopy perturbation algorithm using Laplace transformation, Nonlinear Science Letters A, 1 (2010) 263-267.

17. H.Aminikhah, The combined Laplace transform and new homotopy perturbation methods for stiff systems of ODEs, Applied Mathematical Modelling, 36(8) (2012) 3638-3644.

18. S.C.Sharma and R.K.Bairwa, A reliable treatment of iterative Laplace transform method for fractional telegraph equations, Annals of Pure and Applied Mathematics, 9(1) (2014) 81-89.

19. M.Ramesh Kumar and G.Uthra, A study on numerical stability of finite difference formulae for numerical differentiation and integration, Annals of Pure and Applied Mathematics, 8( 2) (2014) 27-36. 